\title{
Glia-to-Neuron Conversion Alleviates Symptoms of Neurological Disease in Mice
}

A recent study published in Cell revealed that gliato-neuron conversion alleviates symptoms of two top-ranked neurological diseases in mice. This work was performed by researchers from Dr. YANG; Hui's Lab at the Center for Excellence in Brain Science and Intelligence Technology. Institute of Neuroscience (ION), State Key Laboratory of Neuroscience. Chinese Academy of Sciences.

This study showed that knockdown of a single gene Ptbpl using a recently developed RNA-targeting CRISPR svstem CasRx, resulted in the conversion of Müller glia (MG) into retinal ganglion cells (RC,Cs) in mature retinas, leading to the restoration of visual responses in a mouse model with permanent vision impairment. Using a similar approach, it demonstrated that knockdown of $P t b p l$ in the striatum locally induced neurons expressing dopaminergic markers with a very high efficiency and alleviated motor dysfunctions in a mouse model of Parkinson's disease (PD). Together, this study provides a new approach for treating a variety of disorders due to neuronal loss.

The human nervous system contains hundreds to thousands of different types of neurons. In the mature nervous system, it is commonly accepted that neurons
AAV-GFAP-CasRx-gRNA
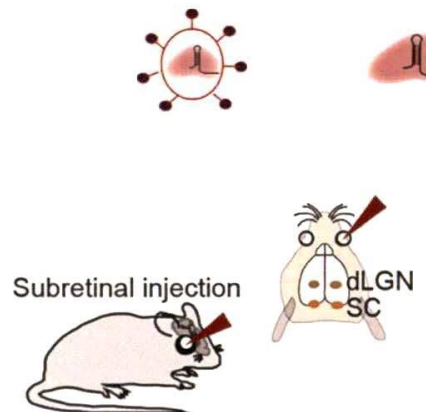

Retinal ganglion cells (RGCs) loss model
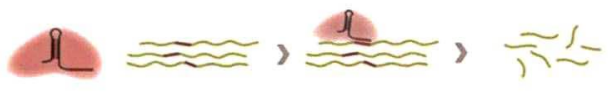

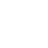


could not proliferate and be regenerated after injury. Thus, the death of neurons may result in the occurrence of different neurodegenerative diseases. such as Nzheimer's disease and PD. Currently. the pathogenesis of these diseases remains unclear and no cure exists.

It was estimated that more than 100 million people worldwide are living with neurodegenerative diseases and the number will increase with the increasing aging population. Among common neurodegenerative disorders, RGC death-induced permanent blindness and PD are two special ones. which are caused by the death of specific types of neurons.

The reason why we could see the outside world is because there is an intact visual pathway between the eve and brain. Retinal ganglion cells (RCCis), the sole output neurons of the retina. are very sensitive to the harmful stimuli. Based on previous reports, RGC degeneration occurs in many ocular diseases such as glaucoma. which is the leading cause of permanent blindness (more than 10 million worldwide). PD, the second most common neurodegenerative disease, is characterized by the progressive death of dopamine neurons in the substantia nigra. leading to a reduction of dopamine concentration in the striatum. It was estimated that close to 10 million people are affected by this disease globally and nearly half of the population are in China. Regeneration of these two specific types of neruons in the adult nervous system is a world-class problem and the aim of many scientists.

To specifically downregulate Ptbp/ expression in the retinal MG with a high efficiency. the authors first screened six guide RNAs (gRNAs) for their efficiency in CasRx editing of $P(b) /$ in both N2a cells and cultured astrocytes, and designed a system to specifically label MG and express CasRx in MG. The vectors were packaged into $\mathrm{Al} / \mathrm{s}$ and introduced to the mature retinas by subretinal injection. One month after subretinal injection. the researchers found many RGCis derived from MG in retinal ganglion cell layer (C.CL). Like normal RCCC. converted RGCis showed action potentials in response to light stimulation and established central projection in the brain and relayed visual information to the dLCN neurons. The function of central projections of MG;-derived RC:Cis was further examined by showing that CasRx-mediated conversion of MC to RGCis could restore vision-dependent behavior that was lost by NMDA-induced retinal injury. To generalize the potential usefulness of CasRx-induced glia-to-neuron conversion in other systems, the researcher further examined whether knockdown of Ptlpl in the striatum could locally convert other types of cells into dopamine neurons, an approach that may be useful for replenishing dopamine in the straitum due to degeneration of dopaminergic neurons in midbrain substantia nigra associated with PD. The researcher found that knockdown of Ptbpl specifically converted striatal astrocytes into neurons with dopaminergic features with a high efficiency and alleviated motor dysfunctions in PD mice.

Although this is an important breakthrough, it should be noted that many important questions have not been addressed yet. Could human RC, (is be induced or could patients with PD be cured using this approach? To answer these questions, it requires the efforts of many scientists in the future.

\section{Contact}

YANG Hui

Center for Excellence in Brain Science and Intelligence Technology, Institute of Neuroscience, Chinese Academy of Sciences Email: huiyang@ion.ac.cn

\section{Reference}

Haibo Zhou, Jinlin Su, Xinde Hu, Changyang Zhou, He Li, Zhaorong Chen, Qingquan Xiao, Bo Wang, Wenyan Wu, Yidi Sun, Yingsi Zhou, Cheng Tang, Fei Liu, Linhan Wang, Canbin Feng, Mingzhe Liu, Sanlan Li, Yifeng Zhang, Huatai Xu, Haishan Yao, Linyu Shi, Hui Yang*, (2020) Glia-toneuron conversion by CRISPR-CasRx alleviates symptoms of neurological disease in mice. Cell. doi:10.1016/j.cell.2020.03.024. 